\title{
Perfil Sociodemográfico, Delictivo y Psicopatológico en una Muestra de Mujeres en Prisión
}

\author{
Sociodemographic, Criminal and Psychopathological Profile in a Sample of Women \\ in Prison
}

\author{
Verónica Molina-Coloma ${ }^{1}$, José Ignacio Pérez ${ }^{2}$ y Karmele Salaberría ${ }^{3}$
}

\section{Resumen}

El objetivo principal de este trabajo es establecer un perfil sociodemográfico, delictivo y psicopatológico de una muestra de mujeres en prisión. La muestra la componen 50 mujeres y 51 hombres de un centro penitenciario en Ecuador. Los resultados sugieren que las mujeres presentan más síntomas de somatización, depresión, ansiedad fóbica e ideación paranoide, puntuaciones más altas en los patrones clínicos de personalidad esquizoide y depresiva que los hombres. En las mujeres se puede observar que aquellas con antecedentes de ingresos en centros juveniles y con mayor número de experiencias traumáticas, presentan más síntomas psicopatológicos y puntuaciones altas en los patrones de personalidad clínica. En conclusión, se considera oportuno establecer protocolos de intervención específicos para las mujeres recluidas en prisión.

Palabras clave: mujeres, prisión, psicopatología, personalidad, Ecuador

\begin{abstract}
The main objective of this research is to establish a sociodemographic, crime and psychopathological profile of women in prison. The sample consists of 50 women and 51 men prisoners in Ecuador. The results suggest that women have more symptoms of somatization, depression, phobic anxiety and paranoid ideation, higher scores in schizoid and depressive personality clinical patterns than men. In women, it can be observed that those with a history of admissions in juvenile centers and with a greater number of traumatic experiences present more psychopathological symptoms and high scores in the clinical personality patterns. In conclusion, the results indicate the psychological needs of women in prison and the importance of considering the differences between men and women to carry out intervention programs.
\end{abstract}

Keywords: women, prison, psychopathology, personality, Ecuador

Agradecimientos: Convenio de Cooperación Interinstitucional entre la Universidad Técnica de Ambato y el Ministerio de Justicia, Derechos Humanos y Cultos, que permitió el acceso al centro penitenciario de Ambato en Ecuador.

\footnotetext{
${ }^{1}$ Doctora. Profesora Titular. Universidad Autónoma de Coahuila. Facultad de Psicología, Unidad Camporredondo, 25020 Saltillo-Coahuila, México. Tel.: 6672325755. Correo: veronicamolinacoloma@gmail.com (Autora de correspondencia)

${ }^{2}$ Doctor. Profesor Titular. Universidad del País Vasco. Facultad de Psicología, Avda. Tolosa, 70, 20018, San Sebastián, España. Tel.: 943015641. Correo: joseignacio.perez@ehu.eus

${ }_{3}^{3}$ Doctora. Profesora Titular. Universidad del País Vasco. Facultad de Psicología, Avda. Tolosa, 70, 20018, San Sebastián, España. Tel.: 943015635. Correo: mcarmen.salaberria@ehu.eus 


\section{Introducción}

Las mujeres en prisión frente a los hombres en la mayoría de los países constituyen una proporción muy pequeña de la población de recluidos, a pesar de que en estos últimos años ha crecido el número de mujeres recluidas, los porcentajes siguen siendo menores a comparación a los hombres (JuanateyDorado, 2018). En mayor medida las mujeres se encuentran cumpliendo una condena por cometer delitos menores. Sin embargo, es importante resaltar también que en estos últimos años se ha observado un aumento de la participación de mujeres en la comisión de delitos violentos (Loinaz, 2016; Van den Bergh et al., 2011).

En España, por ejemplo, la cifra de mujeres representa el $7.48 \%$ de la población total de recluidos mientras que el porcentaje en la población masculina representa el $92.52 \%$ (Juanatey-Dorado, 2018). En América del Sur, el porcentaje de mujeres recluidas en las cárceles es de alrededor del 6\%, concretamente los países que más número de mujeres en prisión albergan en esta zona geográfica son, Bolivia con el $13.4 \%$ y Ecuador con el 10.7\% (Giacomello, 2013). Sin embargo, en el contexto ecuatoriano esta cifra ha decrecido al $8.1 \%$ (Ministerio de Justicia, Derechos Humanos y Cultos [MJDHC] y Comité Internacional de la Cruz Roja [CICR], 2014).

Los delitos por los cuales se encuentran recluidas las mujeres en América del Sur son los delitos relacionados con el microtráfico de drogas, es decir, cerca del $70 \%$ tal es el caso de países como Argentina, Brasil y Costa Rica, donde más del $60 \%$ de las mujeres están privadas de libertad por este tipo de delitos, y en Ecuador llegan al 80\% (Espinoza, 2016). En general las mujeres suelen cometer con más frecuencia delitos contra la propiedad y tráfico de drogas (Espinoza, 2016; Harrison \& Norton-Hawk, 2010).

Respecto a los problemas de salud mental, las mujeres encarceladas presentan mayores niveles de psicopatología a diferencia de las personas pertenecientes a la población general, a las mujeres de la comunidad y a los hombres encarcelados (Álvarez-Pascual \& Jenaro, 2016; Collier \& Friedman, 2016; Gunter et al., 2012; Hales et al., 2016; King et al., 2018; MolinaColoma et al., 2018a; Molina-Coloma et al., 2018b).
Es así, que es frecuente observar, por una parte, un mayor número de síntomas psicopatológicos, trastornos como la depresión mayor, el abuso y la dependencia al alcohol y las drogas, trastorno de estrés postraumático y trastornos de personalidad como el antisocial y el límite en las mujeres recluidas en prisión a diferencia de las mujeres de la población general (Collier \& Friedman, 2016; Molina-Coloma et al., 2018a; Molina-Coloma et al., 2018b). Por otra parte, en comparación con los hombres encarcelados, las mujeres encarceladas tienden a presentar mayores niveles de estrés psicológico, mayor ansiedad, somatización y manía (Drapalski et al., 2009).

En concreto, la presencia de problemas de salud mental de las mujeres encarceladas, por un lado, puede identificarse antes del ingreso a prisión y, por otro lado, durante su estancia en prisión. En este sentido, es importante resaltar que muchas mujeres en situación de reclusión tienen un historial de necesidades sociales, educativas, sanitarias y económicas no cubiertas, además de una historia de violencia familiar y sexual. Los trastornos afectivos y los problemas de comportamiento presentado por las mujeres suelen estar asociados con sentimientos de pérdida e inadecuación (Gunter et al., 2012; Hales et al., 2016; King et al., 2018).

Es así, como este estudio es particularmente necesario. Por una parte, a nivel contextual es relevante, por los pocos estudios realizados en población penitenciaria femenina en Latinoamérica. Asimismo, este estudio aporta a uno de los objetivos del Plan Nacional del Buen Vivir. Este objetivo, atiende al derecho a la rehabilitación social de los jovenes y adultos encarcelados (Consejo Nacional de Planificación [CNP], 2016).

Por otra parte, este estudio es relevante también, porque en el caso de las mujeres, las experiencias adversas tempranas como ser víctimas de abusos físicos y sexuales, los roles sociales de mujeres, algunas formas autodestructivas y mecanismos de afrontamiento a las demandas y dificultades pueden crear necesidades de salud mental específicas de género en prisión (Bartlett \& Hollins, 2018). En ese sentido, este estudio permitiría dar pautas para una intervención psicológica adecuada a las 
necesidades de las mujeres encarceladas (De Vogel \& Nicholls, 2016).

Tomando en consideración lo mencionado anteriormente el presente estudio plantea la siguiente pregunta: ¿Cuál es el perfil sociodemográfico, delictivo y psicopatológico de una muestra de mujeres en prisión en Ecuador?

\section{Método}

\section{Diseño}

Se ha realizado un estudio descriptivocomparativo de corte transversal en una muestra de personas encarceladas en un centro penitenciario ubicado en Ambato-Ecuador.

\section{Participantes}

Los criterios de inclusión para el estudio fueron los siguientes: a) tener capacidad para leer y escribir, b) dar el consentimiento para participar en el estudio, c) no estar en prisión preventiva, y d) presentar perfiles válidos en el MCMI-III.

De las 80 mujeres recluidas en prisión, 50 cumplieron los criterios de inclusión y se compararon con un grupo de 51 hombres recluidos procedentes de la misma prisión.

\section{Instrumentos}

Cuestionario de características sociodemográficas y de salud. El cuestionario fue realizado "ad hoc" por la primera autora del estudio. El cuestionario recopilaba información relacionada a características sociodemográficas. Estuvo conformado por seis preguntas cerradas a excepción de edad, un grupo de preguntas eran de tipo dicotómicas y otras preguntas incluían varias alternativas de respuesta. La información obtenida fue contrastada con la existente en el centro penitenciario.

Cuestionario de características delictivas. El cuestionario fue realizado "ad hoc" por la primera autora del estudio. El cuestionario recopilaba información relacionada con el delito. Estuvo conformado por siete preguntas cerradas, a excepción de tiempo en prisión, un grupo de preguntas eran de tipo dicotómicas y otras preguntas incluían varias alternativas de respuesta. La información obtenida fue contrastada con la existente en el centro penitenciario.
Cuestionario de 90 Síntomas Revisado (SCL-90R). Se utilizó la versión en castellano de González de Rivera et al. (2002). Es un cuestionario de 90 ítems que se agrupan en 9 dimensiones que evalúan malestar psicológico. Cada ítem presenta cinco alternativas de respuesta que oscilan entre 0 (nada) y 4 (mucho). Las dimensiones del cuestionario son: somatización, obsesión-compulsión, sensibilidad interpersonal, depresión, ansiedad, hostilidad, ansiedad fóbica, ideación paranoide, psicoticismo y una escala de ítems adicionales de relevancia clínica. Además, presenta tres índices generales, que reflejan el nivel de gravedad global de la sintomatología del sujeto: Índice General Sintomático (IGS), Total de Síntomas Positivos (TSP) e Índice de Malestar Sintomático Positivo (PSDI). González de Rivera et al. (2002) señalan que este instrumento presenta una consistencia interna alta, con valores de coeficiente alfa, que oscilan entre .78 y .90 . En la población ecuatoriana este instrumento presenta un alfa de Cronbach de .95 (Molina-Coloma, et al., 2018).

\section{Cuestionario para Experiencias Traumáticas} (TQ). Se utilizó la versión en castellano de Bobes et al. (2000). Es un instrumento que evalúa experiencias traumáticas. El cuestionario inicialmente consta de 18 ítems, es un listado de experiencias traumáticas, a las que el sujeto tiene que responder si las ha sufrido o no alguna vez en su vida, y en caso afirmativo, a qué edad sufrió el acontecimiento y durante cuánto tiempo. La segunda parte, evalúa las características del acontecimiento elegido por el sujeto como más importante y explora los síntomas del TEPT. Los ítems son de respuesta dicotómica (sí o no).

El coeficiente alfa es .99 para la escala completa, .97 para la subescala de frecuencia de acontecimientos traumáticos, y .98 para la subescala de severidad (Bobes et al., 2000). En población penitenciaria ecuatoriana este instrumento presenta un alfa de Cronbach de .87 (Molina-Coloma et al., 2021).

Inventario clínico multiaxial de Millon-III (MCMI-III). Se trabajó con la versión en castellano de Cardenal y Sánchez (2007). El inventario evalúa 14 patrones clínicos de la personalidad (esquizoide, evitativa, depresiva, dependiente, histriónica, narcisista, antisocial, agresiva-sádica, compulsiva, negativista, autodestructiva, esquizotípica, paranoide y límite) 
Tabla 1. Comparación en características sociodemográficas y delictivas entre hombres y mujeres

\begin{tabular}{|c|c|c|c|c|c|c|c|}
\hline \multirow{3}{*}{$\begin{array}{l}\text { Características } \\
\text { Sociodemográficas y del delito }\end{array}$} & \multirow{2}{*}{\multicolumn{2}{|c|}{$\begin{array}{c}\text { Mujeres } \\
n=50 \\
\end{array}$}} & \multirow{2}{*}{\multicolumn{2}{|c|}{$\begin{array}{c}\text { Hombres } \\
n=51\end{array}$}} & \multirow[b]{3}{*}{$t$} & \multirow[b]{3}{*}{$p$} & \multirow[b]{3}{*}{$d$} \\
\hline & & & & & & & \\
\hline & $M$ & $D T$ & $M$ & $D T$ & & & \\
\hline Edad & 33.84 & 8.63 & 34.10 & 11.02 & .131 & .896 & .026 \\
\hline \multirow{2}{*}{ Tiempo de estancia en prisión } & 18.66 & 18.96 & 32.90 & 36.17 & 2.49 & .015 & .049 \\
\hline & $n$ & $\%$ & $n$ & $\%$ & $X^{2}$ & $p$ & $V$ \\
\hline Estado Civil & & & & & 1.88 & .598 & .136 \\
\hline Soltera & 18 & 36 & 16 & 31.37 & & & \\
\hline Casada/ Unión libre & 26 & 52 & 24 & 47.06 & & & \\
\hline Separada/Divorciada & 5 & 10 & 8 & 15.69 & & & \\
\hline Viuda & 1 & 2 & 3 & 5.88 & & & \\
\hline Hijos & & & & & 3.01 & .083 & .173 \\
\hline $\mathrm{Si}$ & 44 & 88 & 38 & 74.51 & & & \\
\hline No & 6 & 12 & 13 & 25.49 & & & \\
\hline Ingresos familiares & & & & & 1.10 & .295 & .104 \\
\hline 340 o menos (dólares) & 38 & 76 & 43 & 84.31 & & & \\
\hline 341 o más (dólares) & 12 & 24 & 8 & 15.69 & & & \\
\hline Trabajo antes del ingreso & & & & & 5.49 & 0.02 & 0.23 \\
\hline $\mathrm{Si}$ & 39 & 78 & 48 & 94.12 & & & \\
\hline No & 11 & 22 & 3 & 5.88 & & & \\
\hline Trabajo en la cárcel & & & & & 2.93 & 0.87 & 0.17 \\
\hline $\mathrm{Si}$ & 18 & 36 & 27 & 52.94 & & & \\
\hline No & 32 & 64 & 24 & 47.06 & & & \\
\hline Reincidente & & & & & .241 & .624 & .049 \\
\hline $\mathrm{Si}$ & 24 & 48 & 22 & 43.14 & & & \\
\hline No & 26 & 52 & 29 & 56.86 & & & \\
\hline Tipología delictiva & & & & & 22.99 & .000 & .477 \\
\hline Delitos contra la vida & 3 & 6 & 7 & 13.73 & & & \\
\hline Delitos sexuales & 1 & 2 & 10 & 19.61 & & & \\
\hline Delitos contra la propiedad & 18 & 36 & 25 & 49.02 & & & \\
\hline Delitos de estupefacientes & 21 & 42 & 4 & 7.84 & & & \\
\hline Asociación ilícita & 6 & 12 & 3 & 5.88 & & & \\
\hline Otros delitos & 1 & 2 & 2 & 3.92 & & & \\
\hline Centro de menores infractores & & & & & .509 & .476 & .071 \\
\hline $\mathrm{Si}$ & 17 & 34 & 14 & 27.45 & & & \\
\hline No & 33 & 66 & 37 & 72.55 & & & \\
\hline Familiar en prisión & & & & & 7.32 & .007 & .269 \\
\hline $\mathrm{Si}$ & 34 & 68 & 21 & 41.18 & & & \\
\hline No & 16 & 32 & 30 & 58.82 & & & \\
\hline Familiar en prisión & & & & & 23.23 & .000 & .480 \\
\hline Nadie & 16 & 32 & 30 & 58.82 & & & \\
\hline Familia de origen & 15 & 30 & 9 & 17.65 & & & \\
\hline Familia propia & 12 & 24 & 2 & 3.92 & & & \\
\hline Familia extensa & 2 & 4 & 10 & 19.61 & & & \\
\hline Mixta & 5 & 10 & 0 & 0 & & & \\
\hline Recibe visitas & & & & & 1.54 & .215 & .123 \\
\hline $\mathrm{Si}$ & 36 & 72 & 42 & 82.35 & & & \\
\hline No & 14 & 28 & 9 & 17.65 & & & \\
\hline
\end{tabular}

y 10 síndromes clínicos (trastorno de ansiedad, somatomorfo, bipolar, distimico, dependencia de alcohol y sustancias, trastorno de estrés postraumático, trastorno del pensamiento, depresión mayor y trastorno delirante).

Asimismo, el cuestionario presenta cuatro índices para el control de las respuestas: la escala de validez (que consta de tres ítems y que respondiendo a dos o más se considera un perfil invalido) y las escalas de sinceridad, deseabilidad social y devaluación. Consta de 175 ítems, que se responden como verdadero o falso. Presenta un alfa de Cronbach entre .82 y .96. En estudios anteriores en población penitenciaria ecuatoriana, el alfa de Cronbach fue de .92 (Molina-Coloma et al., 2018).

\section{Procedimiento}

Después de obtener el permiso para el acceso al centro penitenciario, las mujeres y los hombres recluidos recibieron una carta informativa con las características y el objetivo del estudio. Aquellos 
Tabla 2. Comparación entre hombres y mujeres en sintomatología psicopatológica en el SCL-90-R

\begin{tabular}{|c|c|c|c|c|c|c|c|c|c|}
\hline \multirow[t]{2}{*}{ SCL-90-R } & \multicolumn{3}{|c|}{$\begin{array}{c}\text { Mujeres } \\
n=50\end{array}$} & \multicolumn{3}{|c|}{$\begin{array}{c}\text { Hombres } \\
n=51\end{array}$} & \multirow[b]{2}{*}{$t$} & \multirow[b]{2}{*}{$p$} & \multirow[b]{2}{*}{$d$} \\
\hline & $M$ & $D T$ & $T$ & $M$ & $D T$ & $T$ & & & \\
\hline Somatización & 1.66 & .97 & 63 & 0.91 & .85 & 63 & 4.12 & .000 & 0.82 \\
\hline Obsesivo-Compulsivo & 1.47 & .88 & 63 & 1.26 & .74 & 63 & 1.26 & .212 & 0.26 \\
\hline Sensibilidad Interpersonal & 1.30 & .96 & 67 & 0.97 & .65 & 63 & 1.98 & .051 & 0.40 \\
\hline Depresión & 1.85 & .81 & 67 & 1.22 & .79 & 63 & 3.99 & .000 & 0.79 \\
\hline Ansiedad & 1.23 & .96 & 63 & 1.05 & .91 & 63 & .980 & .329 & 0.19 \\
\hline Hostilidad & 1.14 & .20 & 60 & 1.20 & .42 & 63 & .226 & .822 & 0.05 \\
\hline Ansiedad Fóbica & 1.02 & .99 & 63 & 0.62 & .68 & 63 & 2.35 & .021 & 0.47 \\
\hline Ideación Paranoide & 1.55 & .07 & 67 & 1.15 & .85 & 63 & 2.11 & .038 & 0.41 \\
\hline Psicoticismo & 1.03 & .91 & 67 & 0.92 & .63 & 67 & .671 & .504 & 0.14 \\
\hline Índice de Gravedad Global & 1.42 & .78 & 67 & 1.03 & .61 & 67 & 2.75 & .007 & 0.56 \\
\hline Total de Síntomas Positivos & 47.98 & .89 & 63 & 41.78 & .39 & 63 & 1.72 & .089 & 0.34 \\
\hline Índice de Malestar Sintomático Positivo & 2.53 & .66 & 63 & 2.17 & .66 & 58 & 2.72 & .008 & 0.55 \\
\hline
\end{tabular}

Nota . $\mathrm{T}=$ puntuación tipificada del baremo de la población general

que accedieron a participar firmaron una hoja de consentimiento informado. La evaluación se realizó individualmente por la primera autora del estudio en la oficina de evaluación y diagnóstico psicológico de la institución. Finalmente, se les ofreció un informe de los resultados a todos los participantes que así lo solicitaron.

\section{Análisis estadísticos}

Se utilizó el paquete estadístico SPSS (versión 22.0) para los respectivos análisis. Se llevaron a cabo análisis descriptivos, análisis de comparación de grupos, según el sexo, para lo cual se utilizó una $t$ de Student y se calculó el tamaño de las diferencias entre los grupos por medio de la $d$ de Cohen. Finalmente, se realizaron análisis de correlaciones, se utilizó la $\mathrm{r}$ de Pearson, con el grupo de mujeres en prisión, entre síntomas psicopatológicos, características de personalidad, síndromes clínicos, con características sociodemográficas, características del delito y con eventos traumáticos.

\section{Resultados}

\section{Diferencias en características sociodemográficas y del delito}

Respecto a las características sociodemográficas, como puede observarse, no se presentan diferencias estadísticamente significativas entre hombres y mujeres en la mayoría de las características, exceptuando en el trabajo antes del ingreso a prisión, donde el $94.1 \%$ de los hombres trabajaban mientras que, sólo un $78 \%$ de las mujeres lo hacía. Desde el punto de vista delictivo, las mujeres llevan menos tiempo en prisión, han sido recluidas principalmente por delitos contra la propiedad y tenencia de estupefacientes, y tienen a más familiares en prisión, tanto de su familia de origen como de la propia familia.

\section{Diferencias entre mujeres $y$ hombres en síntomas psicopatológicos}

Las mujeres encarceladas presentaron más síntomas psicopatológicos que los hombres encarcelados por encima del punto de corte ( $\mathrm{T}=63$ ), y estas diferencias son estadísticamente significativas en somatización $(d=0.82)$, depresión $(d=0.79)$, severidad global de síntomas $(d=0.56)$, malestar sintomático positivo $(d=0.55)$, ansiedad fóbica $(d=0.47)$, ideación paranoide $(d=0.41)$, y sensibilidad interpersonal $(d=0.40)$.

Diferencias entre mujeres $y$ hombres en patrones clínicos de personalidad y síndromes clínicos

En los patrones clínicos de personalidad evaluados con el MCMI-III desde un punto de vista comparativo entre los hombres y las mujeres, aparecen diferencias en los rasgos de personalidad esquizoide $(d=0.47)$, y depresiva $(d=0.48)$, siendo las mujeres quienes presentaron puntuaciones más altas.

Con respecto a los síndromes clínicos, se presentaron diferencias significativas en depresión mayor $(d=0.69)$, trastorno somatomorfo $(d=0.46)$, $\mathrm{y}$ en dependencia a sustancias $(d=0.39)$. Las puntuaciones más altas en depresión mayor y trastorno somatomorfo correspondían a mujeres, mientras que en los hombres apareció la dependencia a sustancias con mayor puntuación. 
Tabla 3. Comparación entre hombres y mujeres en rasgos de personalidad y síndromes clínicos en el MCMI-III

\begin{tabular}{|c|c|c|c|c|c|c|c|c|c|}
\hline \multirow[t]{2}{*}{ MCMI-III } & \multicolumn{3}{|c|}{$\begin{array}{c}\text { Mujeres } \\
n=50\end{array}$} & \multicolumn{3}{|c|}{$\begin{array}{c}\text { Hombres } \\
n=51\end{array}$} & \multirow[b]{2}{*}{$t$} & \multirow[b]{2}{*}{$p$} & \multirow[b]{2}{*}{$d$} \\
\hline & $M$ & $D T$ & Prev & $M$ & $D T$ & Prev & & & \\
\hline \multicolumn{10}{|l|}{ Patrones clínicos de personalidad } \\
\hline Esquizoide & 9.16 & 2.71 & 63 & 7.80 & 3.02 & 60 & 2.38 & .019 & 0.47 \\
\hline Evitativa & 7.32 & 2.99 & 53 & 7.16 & 3.43 & 60 & .255 & .800 & 0.05 \\
\hline Depresiva & 8.02 & 3.88 & 40 & 6.25 & 3.55 & 45 & 2.38 & .019 & 0.48 \\
\hline Dependiente & 7.16 & 3.63 & 42 & 6.78 & 3.53 & 47 & .527 & .600 & 0.11 \\
\hline Histriónica & 9.46 & 3.21 & 46 & 9.12 & 3.03 & 27 & .551 & .583 & 0.11 \\
\hline Narcisista & 11.28 & 4.80 & 60 & 11.04 & 4.73 & 62 & .254 & .800 & 0.05 \\
\hline Antisocial & 9.46 & 3.91 & 68 & 10.16 & 4.10 & 65 & .874 & .384 & 0.17 \\
\hline Agresiva-Sádica & 10.34 & 5.17 & 63 & 10.53 & 4.42 & 64 & .198 & .844 & 0.04 \\
\hline Compulsiva & 10.90 & 2.78 & 44 & 11.31 & 2.76 & 35 & .751 & .455 & 0.15 \\
\hline Negativista & 9.48 & 4.26 & 64 & 8.61 & 3.80 & 60 & 1.09 & .280 & 0.22 \\
\hline Autodestructiva & 6.22 & 3.59 & 60 & 5.37 & 3.52 & 60 & 1.20 & .234 & 0.24 \\
\hline Ezquizotípica & 6.46 & 3.82 & 61 & 6.18 & 3.93 & 62 & .368 & .714 & 0.07 \\
\hline Límite & 7.62 & 4.43 & 47 & 6.22 & 3.59 & 51 & 1.76 & .082 & 0.35 \\
\hline Paranoide & 9.88 & 3.87 & 67 & 8.92 & 3.90 & 66 & 1.30 & .218 & 0.25 \\
\hline \multicolumn{10}{|l|}{ Síndromes clínicos } \\
\hline Trastorno de ansiedad & 6.70 & 3.86 & 60 & 5.63 & 3.88 & 68 & 1.39 & .167 & 0.28 \\
\hline Trastorno somatomorfo & 5.18 & 3.25 & 43 & 3.75 & 2.99 & 46 & 2.31 & .023 & 0.46 \\
\hline Trastorno bipolar & 6.56 & 3.07 & 60 & 7.12 & 3.13 & 63 & .903 & .369 & 0.18 \\
\hline Trastorno distimico & 6.80 & 3.55 & 45 & 5.45 & 3.55 & 40 & 1.91 & .059 & 0.38 \\
\hline Dependencia alcohol & 6.78 & 3.59 & 72 & 7.04 & 3.23 & 71 & .381 & .704 & 0.08 \\
\hline Dependencia sustancias & 6.40 & 3.77 & 66 & 8.00 & 3.91 & 70 & 2.09 & .039 & 0.42 \\
\hline Trastorno de estrés postraumatico. & 8.24 & 4.82 & 63 & 6.45 & 4.48 & 62 & 1.93 & .056 & 0.38 \\
\hline Trastorno pensamiento & 7.94 & 4.90 & 53 & 6.49 & 4.47 & 51 & 1.55 & .124 & 0.31 \\
\hline Depresión mayor & 8.44 & 5.16 & 60 & 5.16 & 4.28 & 62 & 3.48 & .001 & 0.69 \\
\hline Trastorno delirante & 6.14 & 2.98 & 75 & 5.71 & 2.99 & 77 & .731 & .466 & 0.14 \\
\hline Índice de sinceridad & 100.52 & 33.28 & 55 & 94.51 & $\begin{array}{c}32.9 \\
5\end{array}$ & 51 & .912 & .364 & 0.18 \\
\hline Índice de deseabilidad social & 11.48 & 6.00 & 55 & 10.98 & 3.73 & 53 & .503 & .616 & 0.10 \\
\hline Índice de devaluación & 15.36 & 8.10 & 63 & 13.20 & 8.44 & 63 & 1.32 & .192 & 0.26 \\
\hline
\end{tabular}

Nota. $\mathrm{PREV}=$ puntuaciones de prevalencia

Relación entre síntomas psicopatológicos y características sociodemográficas, delictivas y experiencias traumáticas en mujeres

Las correlaciones se presentaron negativas con la edad y con el tiempo en prisión. Asi, a menor edad en las mujeres encarceladas $(n=50)$ más síntomas psicopatológicos como obsesióncompulsión, sensitividad interpersonal, ansiedad, hostilidad, ansiedad fóbica, ideación paranoide, psicoticismo, mayor severidad global de síntomas y mayor malestar sintomático positivo. Asimismo, las mujeres encarceladas $(n=50)$ que llevaban menos tiempo en prisión presentaban mayor sensitividad interpersonal, ansiedad e ideación paranoide.

Las correlaciones se presentaron positivas con el número de experiencias traumáticas y con el número de experiencias traumáticas interpersonales. Así, a mayor número de experiencias traumáticas mayor sintomatología psicopatológica a excepción de hostilidad y ansiedad fóbica. Del mismo modo, a mayores experiencias traumáticas interpersonales, mayor somatización, obsesión-compulsión, sensitividad interpersonal, depresión, ansiedad, ideación paranoide, psicoticismo, severidad global de síntomas y malestar sintomático positivo.

Relación entre patrones clínicos de la personalidad y síndromes clínicos y características sociodemográficas, delictivas y experiencias traumáticas en mujeres

En el grupo de mujeres $(n=50)$, desde el punto de vista de los patrones clínicos de personalidad y de los síndromes clínicos evaluados con el MCMI-III, se presentaron correlaciones negativas entre la edad y el tiempo en prisión. Así, a menor edad se evidenció una mayor presencia de patrones clínicos de la personalidad y síndromes clínicos. En cuanto a las características delictivas, a menor tiempo en prisión más presencia de patrónes clínicos de personalidad antisocial, agresiva-sádica, compulsiva, esquizotípica, límite, dependencia de alcohol, dependencia de sustancias, trastorno de estrés postraumático, trastorno del pensamiento y trastorno delirante.

Bajo esta misma línea, se presentaron correlaciones positivas en ingresos a prisión y en 
Tabla 4. Correlaciones entre síntomas psicopatológicos, con la edad, características delictivas y experiencias traumáticas

\begin{tabular}{|c|c|c|c|c|c|c|}
\hline & Edad & $\begin{array}{c}\text { Tiempo en } \\
\text { prisión }\end{array}$ & $\begin{array}{l}\text { Historial } \\
\text { Ingresos en } \\
\text { prisión }\end{array}$ & $\begin{array}{c}\text { Historial } \\
\text { Ingresos en el centro } \\
\text { de menores } \\
\text { infractores }\end{array}$ & $\begin{array}{l}\text { Experiencias } \\
\text { traumáticas }\end{array}$ & $\begin{array}{c}\text { Experiencias } \\
\text { traumáticas } \\
\text { interpersonales }\end{array}$ \\
\hline \multicolumn{7}{|l|}{ SCL-90- R } \\
\hline Somatización & -.015 & 0.02 & -.194 & .141 & $.459 * *$ & $431 * *$ \\
\hline Obsesión-Compulsión & $-.426 * *$ & -.189 & -.188 & .143 & $.417 * *$ & $.300 *$ \\
\hline Sensibilidad Interpersonal & $-.378 * *$ & $-.289 *$ & -.089 & .107 & $.392 * *$ & $.310^{*}$ \\
\hline Depresión & -.217 & -.131 & -.097 & .082 & $.367 * *$ & $.297 *$ \\
\hline Ansiedad & $-.382 * *$ & $-.290 *$ & .027 & .200 & $.422 * *$ & $.362 * *$ \\
\hline Hostilidad & $-.280 *$ & -.124 & -.088 & .117 & .248 & .233 \\
\hline Ansiedad Fóbica & $-.346^{*}$ & -.269 & -.053 & .028 & .267 & .206 \\
\hline Ideación Paranoide & $-.408 * *$ & $-.436 * *$ & 0.40 & .110 & $.464 * *$ & $.421 * *$ \\
\hline Psicoticismo & $-.359 *$ & -.253 & -0.82 & .088 & $.480 * *$ & $.426^{* *}$ \\
\hline Índice de Severidad Global & $-.357 *$ & -.249 & -.102 & .140 & $.485^{* *}$ & $.413 * *$ \\
\hline Total de Síntomas Positivos & $-.389 * *$ & -.240 & -.012 & .101 & $.317 *$ & $.318 *$ \\
\hline Índice de Malestar Sintomático Positivo & -.139 & -.110 & -.131 & .248 & $.536 * *$ & $.411 * *$ \\
\hline
\end{tabular}

Nota. $* p<.05 ; * * p<.01$

Tabla 5. Correlaciones entre patrones clínicos de personalidad y síndromes clínicos, con edad, características delictivas y experiencias traumáticas

\begin{tabular}{|c|c|c|c|c|c|c|}
\hline & Edad & $\begin{array}{c}\text { Tiempo en } \\
\text { prisión }\end{array}$ & $\begin{array}{l}\text { Historial de } \\
\text { Ingresos en } \\
\text { prisión }\end{array}$ & $\begin{array}{l}\text { Historial de ingresos en } \\
\text { el centro de menores } \\
\text { infractores }\end{array}$ & $\begin{array}{c}\text { Experiencias } \\
\text { traumáticas }\end{array}$ & $\begin{array}{c}\text { Experiencias } \\
\text { traumáticas } \\
\text { interpersonales }\end{array}$ \\
\hline \multicolumn{7}{|l|}{ Patrones clínicos de personalidad } \\
\hline Esquizoide & -.205 & -.225 & -.133 & .203 & $.346^{*}$ & .245 \\
\hline Evitativa & $-.354 *$ & -.247 & -.169 & .092 & $.331 *$ & .233 \\
\hline Depresiva & $-.409 * *$ & -.232 & -.037 & .180 & $.412 * *$ & $.365^{* *}$ \\
\hline Dependiente & $-.348^{*}$ & -.127 & .006 & .069 & $.396 * *$ & $.314 *$ \\
\hline Histriónica & -.174 & -.027 & -.184 & -.021 & .240 & .212 \\
\hline Narcisista & $-.351 *$ & -.198 & .015 & .097 & $.420 * *$ & $.338 *$ \\
\hline Antisocial & $-.562 * *$ & $-.516^{* *}$ & $.335^{*}$ & $.362 * *$ & $.461 * *$ & $.462 * *$ \\
\hline Agresiva-Sádica & $-.584 * *$ & $-.433 * *$ & .229 & $.305 *$ & $.458 * *$ & $.498 * *$ \\
\hline Compulsiva & $-.345^{*}$ & $-.361 *$ & .132 & $.282 *$ & $.505 * *$ & $.507 * *$ \\
\hline Negativista & $-.406^{* *}$ & -.158 & -.007 & .159 & $.412 * *$ & $.372 * *$ \\
\hline Autodestructiva & $-.385 * *$ & -.236 & -.033 & .102 & .185 & .217 \\
\hline Ezquizotípica & $-.365 * *$ & $-.354 *$ & -.013 & .229 & $.535 * *$ & $.516^{* *}$ \\
\hline Límite & $-.461 * *$ & $-.339 *$ & .116 & .254 & $.510 * *$ & $.493 * *$ \\
\hline Paranoide & $-.299 *$ & -.252 & -.037 & .225 & $.390 * *$ & $.441 * *$ \\
\hline \multicolumn{7}{|l|}{ Síndromes clínicos } \\
\hline Trastorno de ansiedad & $-.331 *$ & -.228 & -.071 & .183 & $.512 * *$ & $.468 * *$ \\
\hline Trastorno somatomorfo & -.120 & .012 & -.205 & .074 & $.308^{*}$ & .226 \\
\hline Trastorno bipolar & $-.367 * *$ & -.261 & .206 & .236 & $.471 * *$ & $.499 * *$ \\
\hline Trastorno distimico & $-.371 * *$ & -.261 & -.002 & .162 & $.456 * *$ & $.402 * *$ \\
\hline Dependencia alcohol & $-.494 * *$ & $-.524 * *$ & .275 & $.336 *$ & $.462 * *$ & $.469 * *$ \\
\hline Dependencia sustancias & $-.467 * *$ & $-.527 * *$ & $.355^{*}$ & $.355^{*}$ & $.391 * *$ & $.358 *$ \\
\hline Trastorno de estrés postraumático & $-.423 * *$ & $-.311 *$ & -.050 & .214 & $.568 * *$ & $.505^{* *}$ \\
\hline Trastorno pensamiento & $-.417 * *$ & $-.305^{*}$ & .061 & .190 & $.497 * *$ & $.440 * *$ \\
\hline Depresión mayor & $-.308 *$ & -.154 & -.095 & .092 & $.374 * *$ & $.298 *$ \\
\hline Trastorno delirante & $-.300 *$ & $-.354 *$ & .018 & .232 & $.469 * *$ & $.458 * *$ \\
\hline
\end{tabular}

ingresos al centro de menores infractores. Asi, a mayor número de ingresos en prisión mayor presencia de personalidad antisocial $y$ dependencia a sustancias. Del mismo modo, a mayor número de ingresos a centros de menores infractores en el pasado mayor presencia de patrones clínicos de la personalidad como: antisocial, agresiva-sádica, compulsiva, dependencia al alcohol y las sustancias.

Por otra parte, las experiencias traumáticas correlacionaron positivamente. Así, a mayor número de experiencias traumáticas, mayor presencia de patrones clínicos de personalidad y de síndromes clínicos, a excepción de los patrones clínicos de personalidad autodestructiva e histriónica. Del mismo modo, a mayor número de experiencias traumáticas interpersonales, mayor presencia de patrones clínicos de personalidad y síndromes clínicos, a excepción de los patrones clínicos de personalidad autodestructiva e histriónica y el trastorno somatomorfo. 


\section{Discusión}

El perfil sociodemográfico de las mujeres recluidas en Ecuador tiene cierta similitud con los perfiles encontrados en otros estudios realizados en recintos penitenciarios. En su mayoría son mujeres jóvenes con una media de edad de 33 años, en su mayoría se encuentran solteras o emparejadas, con hijos a su cargo, con ingresos menores al salario básico, y en un $66 \%$ tienen familiares en prisión, bajo nivel académico y la procedencia de medios sociales deprimidos, lo que convierte a estas mujeres en una población especialmente vulnerable (Mundt et al., 2015; Pulido et al., 2009; Villagra-Lanza et al., 2011).

En relación al perfil delictivo, la media de estancia en prisión es de un año y siete meses, el $48 \%$ es reincidente, y en su mayoría las mujeres han sido sentenciadas por delitos contra la propiedad y por tenencia ilegal de estupefacientes. Desde el punto de vista psicopatológico, las mujeres presentan en general más síntomas, síndromes y patrones de personalidad de carácter afectivo, somático y tendencias paranoides a diferencia de los hombres (Drapalski et al., 2009).

En estas mujeres el ser más joven y el experimentar hechos traumáticos se relaciona con una mayor presencia de síntomas psicopatológicos, síndromes clínicos y patrones clínicos de la personalidad. Estos síntomas podrían justificarse debido a las características contextuales carcelarias y por el tipo de relaciones que se establecen entre las mujeres encarceladas (Ruíz, 2007).

En particular, cuando una persona recién ingresa en prisión e intenta adaptarse al contexto carcelario, el sentirse herida fácilmente, incomprendida, frente al resto de las mujeres encarceladas o pensar que las demás compañeras le quieren hacer daño o abusar de ella, es un pensamiento común. Esta conducta defensiva es quizá reforzada por la necesidad de sobrevivir dentro del ambiente carcelario, por lo que resulta difícil distinguir entre la ideación paranoide situacional o la de carácter más crónico. Sin embargo, en este punto puede ser relevante considerar en futuros estudios, el historial de salud mental y así poder identificar el porcentaje de mujeres que ingresan con psicopatología y realizar un seguimiento de su desarrollo dentro de prisión.
El patrón clínico de personalidad antisocial y dependencia de sustancias destaca en las mujeres con mayor historial de ingresos al centro de menores infractores y a la prisión. En este sentido, es probable que estas mujeres, con un historial largo de dificultades y problemas de conducta, presenten un perfil violento precoz y distinto, a aquellas que cometen actos delictivos únicamente en la vida adulta.

En este estudio, la victimización se ha mostrado como una variable importante asociada con la presencia de síntomas psicopatológicos, trastornos y patrones clínicos de personalidad. Las historias de abuso interpersonal y traumáticas previas al ingreso en prisión suelen ser un factor común en las mujeres encarceladas que en muchas ocasiones no suele ser abordado (Gunter et al., 2012).

En concreto, se ha podido evidenciar en Ecuador, que la venta y el tráfico de estupefacientes, es una actividad que algunas mujeres realizan desde sus hogares, ya que les permite continuar paralelamente desempeñando actividades domésticas en la casa, ya que muchas veces constituyen familias monoparentales pobres que fácilmente se convierten en el perfil ideal para las redes del narcotráfico (Pontón, 2006).También cabe señalar que en algunos casos, el establecimiento de relaciones románticas con criminales se considera un factor potencial para cometer delitos por parte de las mujeres (Mauá \& Baltieri, 2012).

En las prisiones de algunos países europeos y anglosajones existen programas de tratamiento diseñados para mujeres en prisión. Por ejemplo, programas de resolución de problemas para mujeres con delitos no violentos, programas de prevención de adicciones, programas de compromiso y participación, programas de regulación de emociones. Este tipo de programas podrían ser adaptados a la población penitenciaria de este país tomando en consideración las características del contexto (Redondo, 2017).

Este estudio no está exento de limitaciones, como lo es, la muestra, que fue pequeña y recogida en un único centro penitenciario. Sin embargo, estos resultados, puede dar paso a ampliar o a abordar a futuro nuevos estudios como, por ejemplo, el estudio del historial del trauma en este tipo de población. Estos estudios 
son de carácter novedoso y son escasos, se tiene poca información sobre este tema en contextos latinoamericanos.

En concreto los resultados obtenidos en este trabajo pueden sin duda contribuir al diseño de programas que se ajusten en mayor medida a las especificidades de dicho colectivo (FernándezÁlvarez, 2006; Oficina de Naciones Unidas contra la Droga y el Delito [UNODC], 2014).

\section{Referencias}

Álvarez-Pascual, P. A., \& Jenaro, C. (2018). Evaluación de la Identidad del Recluso mediante la Técnica de la Rejilla. Revista Iberoamericana de Diagnóstico y Evaluación - e Avaliação Psicológica, 47(2), 5-20. https://doi.org/10.21865/RIDEP47.2.01

Bartlett, A., \& Hollins, S. (2018). Challenges and mental health needs of women in prison. The British Journal of Psychiatry, 212(3), 134136. https://doi.org/10.1192/bjp.2017.42

Bobes, J., Calcedo-Barba, A., García, M., Francois, M., Rico, E., González, M.P., Bascarán, M.T., Bousoño, M., \& miembros del grupo español de trabajo para el estudio del trastorno de estrés postraumático (2000). Evaluación de las propiedades psicométricas de la versión española de cinco cuestionarios para la evaluación del trastorno de estrés postraumático. Actas Españolas de Psiquiatría, 28(4), 207-218.

Cardenal, V., \& Sánchez, P. (2007). Inventario clínico multiaxial de Millon III. Manual de la adaptación española. TEA ediciones.

Collier, S., \& Friedman, S. (2016). Mental illness among women referred for psychiatric services in a New Zealand Women's prison. Behavioral Sciences \& the Law, 34, 539-550. https://doi.org/10.1002/bs1.2238

Consejo Nacional de Planificación (2016). Plan Nacional de Desarrollo 2017-2021. Toda una Vida de Ecuador.

https://www.planificacion.gob.ec/wpcontent/u ploads/downloads/2017/10/PNBV-26-OCT-

FINAL_0K.compressed1.pdf

De Vogel, V., \& Nicholss, T. 1. (2016). Gender matters: An introduction to the special issues on women and girls. International Journal of
Forensic Mental Health, 15(1), 1-25. https://doi.org/10.1080/14999013.2016.11414 39

Drapalski, A., Youman, J., Stuewig, F., \& Tangney, J. (2009). Gender differences in jail inmates' symptoms of mental illness, treatment history and treatment seeking. Criminal Behaviour and Mental Health, 19, 193-206. https://doi.org/10.1002/cbm.733

Espinoza, O. (2016). Mujeres privadas de libertad: ¿Es posible su reinserción social?, Cuaderno CRH, 29(3), 93-107.

Fernández- Álvarez, H. (2006). Conferencia del V Congreso Iberoamericano de Evaluación Psicológica. Los múltiples rostros de la evaluación en psicoterapia. Revista Iberoamericana de Diagnóstico y Evaluación - e Avaliação Psicológica, 1(21), 149-168.

Giacomello, C. (2013). Mujeres, delitos de drogas y sistemas penitenciarios en América Latina. International Drug Policy Consortium.

González de Rivera, J. L., De las Cuevas, C., Rodríguez Abuín, M., \& Rodríguez Pulido, F. (2002). Versión española del SCL-90-R. TEA ediciones.

Gunter, T. D., Chibnall, J. T., Antoniak, S. K., McCormick, B., \& Black, D. W. (2012). Relative contributions of gender and traumatic life experience to the prediction of mental disorders in a sample of incarcerated offenders. Behavioral sciences \& the law, 30(5), 615-630.

https://doi.org/10.1002/bsl.2037

Hales, H., Somers, N., Reeves, C., \& Bartlett, A. (2016). Characteristics of women in a prison mental health assessment unit in England and Wales (2008-2010). Criminal Behaviour and Mental Health, 26, 136-152. https://10.1002/cbm.1953

Harrison, J., \& Norton-Hawk, M. (2010). The care, custody, and control of incarcerated women in Ecuador. Societies Without Borders, 5(1), 21-48.

Juanatey-Dorado, C. (2018). Delincuencia y población penitenciaria femeninas: Situación actual de las mujeres en prisión en España. Revista Electrónica de Ciencia Penal y Criminología, 20(10), 1-32.

King, E., Tripodi, S., \& Veeh, C. (2018). The relationship between severe mental disorders 
and recidivism in a sample of women released from prison. Psychiatric Quarterly, 89, 717731.

https://doi.org/10.1007/s11126-018-9572-9

Loinaz, I. (2016). Cuando "el" delincuente es "ella": Intervención con mujeres violentas. Anuario de Psicología Jurídica, 26, 41-50. https://doi.org/10.1016/j.apj.2016.04.006

Mauá, F. H. N., \& Baltieri, D. A. (2012). Criminal career-related factors among female robbers in the state of Sao Paulo, Brazil, and a presumed 'revolving-door'situation. Revista Brasileira de Psiquiatría, 34(2), 176-184. https://10.1590/s1516-44462012000200010

Ministerio de Justicia, Derechos Humanos y Cultos y Comité Internacional de la Cruz Roja (2014). Manual Derechos de Humanos, aplicado al contexto penitenciario. Grafilyon.

Molina-Coloma, V., Pérez, J. I., \& Salaberría, K., (2018a). Diferencias generales y entre sexos en salud mental: Un estudio comparativo entre población penitenciaria y población general. Revista Mexicana de Psicología, 35(2), 117130.

Molina-Coloma, V., Salaberría, K., \& Pérez, J. I. (2018b). La personalidad en población carcelaria: Un estudio comparativo en Ecuador. Anuario de Psicología Jurídica, 28, 1-7. https://10.5093/apj2018a5

Molina-Coloma, V., Salaberría, K., Pérez, J. I., \& Kendall-Tackett, K. (2021). Traumatic events, psychological symptoms and aggression in male and female prisoners. Psychological Trauma: Theory, Research, Practice, and Policy. Avance en línea. https://10.1037/tra0001039

Mundt, A. P., Kastner, S., Mir, J., \& Priebe, S. (2015). Did female prisoners with mental disorders receive psychiatric treatment before imprisonment? BMC psychiatry, 15(1), 5. https://doi.org/10.1186/s12888-015-0387-z

Oficina de Naciones Unidas contra la Droga y el Delito (2014). Handbook on women and imprisomment. United Nations Office on drugs and crime.

Pontón, J. (2006). Mujeres que cruzaron la línea: Vida cotidiana en el encierro. Facultad Latinoamericana de Ciencias Sociales.

Pulido, F., Rodríguez, J. F., \& Colorado, M. P. (2009). Factores sociodemográficos asociados con los síntomas depresivos en una muestra de mujeres recluidas en dos prisiones de México. Rev Panam Salud Publica, 26(3), 209-215.

Redondo, S. (2017). Evaluación y tratamiento de delincuentes. Jóvenes y adultos. Pirámide.

Ruiz, J. I. (2007). Síntomas psicológicos, clima emocional, cultura y factores psicosociales en el medio penitenciario. Revista Latinoamericana de Psicología, 39(3), 547561.

Van den Bergh, B.J., Gatherer, A., Fraser, A., \& Moller, L. (2011). Imprisonment and women's health: Concerns about gender sensitivity, human rights and public health. Bull World Health Organ, 89(9), 689-694. http://dx.doi.org/10.2471/BLT.10.082842

Villagra-Lanza, P., Gonzáles-Menéndez, A., Fernández-García, P., Casares, M. J., MartínMartín, L., \& Rodríguez-Lameras, F. (2011). Perfil adictivo, delictivo y psicopatológico de una muestra de mujeres en prisión. Adicciones, 23(3), 219-226. 